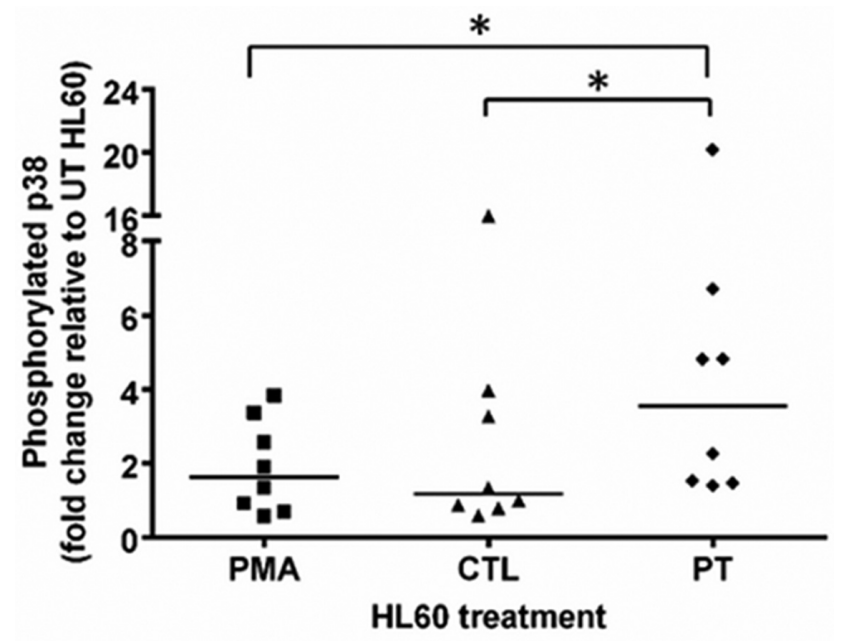

$\mathrm{n}=8 ; *$ denotes $\mathrm{p} \leq \mathbf{0 . 0 5}$ compared to patient (PT) PMP

Abstract 115 Figure 3 Patients PMPs induced significantly higher phosphorylated 38 expression in HL60 cells. HL60 cells were treated with control (CTL) or patient (PT) PMPs prior to cell lysis and western blot analysis for protein p38 phosphorylation.Untreated HL60 and PMAactivated HL60 were used as baseline and activated controls respectively. Phosphorylated p38 bands were quantified using using Image J software and normalised to that of the untreated condition. $n=8$ for each treatment condition.The intensity of phosphorylated p38 bands in PT PMP-treated-HL60 cells was significant higher than that of CTL PMP-treated $(p=0.4)$ and PMA-activated $(p=0.04)$ HL60 cells using Mann-Whitney test.

\section{APOPTOSIS: INITIATION AND PROPAGATION IN THE KINETIC PROCESS OF SYSTEMIC LUPS ERYTHEMATOSUS}

${ }^{1} \mathrm{~L} \mathrm{Na*}$, ${ }^{1} \mathrm{~F}$ Hao. 'Southwest Hospital Third Military Medical University, Dermatology, Chongqing, China

\subsection{6/lupus-2017-000215.116}

Background and aims Apoptosis is a genetically conserved pathway and a regular feature in normal tissue homeostasis. Aberrant cell apoptosis could result in imbalanced immune regulation and plays an important role in the development of autoimmune diseases, especially in systemic lupus erythematosus(SLE).

Methods SLE is a complex,multisystem autoimmune disease characterised by production of high-titer autoantibodies directed against tissue-specific and ubiquitously expressed selfantigens. There has been greatly confirmed that accelerated cell apoptosis and apoptotic debris could account for the accumulation of a great deal of nuclear antigens, which could be presented to antigen presenting cells and lymphocytes, and subsequently induce incomplete immune tolerance,potentially through abnormal apoptotic signalling pathways and abnormal signalling thresholds on responding lymphocytes.Impaired clearance of apoptotic cells is also likely to be an important factor in SLE pathogenesis, which is originated from morphIncreasedological abnormalities, impaired differentiating and adhesion ability of macrophage cells, abnormal chemokines and phagecytosis-related receptors expression and lack of complements.
Results The dysfunction of apoptosis may be a direct consequence of alterations in genes such as Fas, Bcl-2 and C1q. Increased expression of Fas antigen could intensify the exposure of hidden antigens. The overexpression of $\mathrm{Bcl}-2$ protein might inhibit the removal of auto-reactive cells, and the lack of $\mathrm{C} 1 \mathrm{q}$ could impair the clearance of self-antigens.Increased apoptosis of lymphcytes especially regulatory $\mathrm{T}$ cells is also an important reason to lead to breakdown of immuntolerance.

Conclusions The complete knowledge of the role of apoptosis components in the etiopathogenesis of lupus could lead to the development of new therapies targeting the apoptotic threshold, which could result in a more specific and effective disease response compared to global immunosuppression.

\section{MUCOSAL-ASSOCIATED INVARIANT T CELL DEFICIENCY IN SYSTEMIC LUPUS ERYTHEMATOSUS IS RELATED TO TO AN INTRINSIC DEFECT IN THE CA2+/CALCINEURIN/ NFAT1 SIGNALLINGPATHWAY}

${ }^{1}$ YW Park, ${ }^{1} \mathrm{YN}$ Cho, ${ }^{1} \mathrm{HM}$ Jin, ${ }^{2} \mathrm{~S} J$ Kee. ${ }^{1}$ Chonnam National University Hospital, Rheumatology, Gwangju, Republic of Korea; ${ }^{2}$ Chonnam National University Hospital, Laboratory Medicine, Gwangju, Republic of Korea

\subsection{6/lupus-2017-000215.117}

Background and aims Mucosal-associated invariant T (MAIT) cells contribute to protection against certain microorganism infections and play an important role in mucosal immunity. Here, we examined the level and function of MAIT cells in patients with rheumatic diseases.

Methods Patients with systemic lupus erythematosus (SLE; $\mathrm{n}=54$ ), rheumatoid arthritis (RA; $\mathrm{n}=66$ ), Behçet's disease $(n=9)$, ankylosing spondylitis $(n=21)$, and healthy controls $(n=136)$ were enrolled in the study. MAIT cell, cytokine and programmed death-1 (PD-1) levels were measured by flow cytometry.

Results Circulating MAIT cell levels were significantly reduced in SLE and RA patients. This MAIT cell deficiency was more prominent in $\mathrm{CD} 8+$ and double-negative $\mathrm{T}$ cell subsets, and significantly correlated with disease activity, such as SLEDAI and DAS28. Interestingly, MAIT cell frequency was significantly correlated with natural killer T (NKT) cell frequency in SLE patients. IFN- $\gamma$ in MAIT cells was impaired in SLE patients, which was due to an intrinsic defect in the $\mathrm{Ca} 2+/ \mathrm{cal}-$ cineurin/NFAT1 signalling pathway. In SLE patients, MAIT cells were poorly activated by alpha-galactosylceramide-stimulated NKT cells, thereby showing the dysfunction between MAIT cells and NKT cells. Notably, an elevated expression of PD-1 in MAIT cells and NKT cells was associated with SLE. In RA patients, MAIT cell levels were significantly higher in synovial fluid than in peripheral blood.

Conclusions Our study primarily demonstrates that MAIT cells are numerically and functionally deficient in SLE. In addition, we report a novel finding that this MAIT cell deficiency is associated with NKT cell deficiency and elevated PD-1 expression. 